\title{
Acute Cardiac Tamponade Secondary to Cardiac Vascular-Type Pleomorphic Leiomyosarcoma: Case Report
}

\author{
Ricardo Fernández-Ferreira ${ }^{a}$ Pamela Denisse Soberanis-piña ${ }^{a}$ \\ Emilio Conde-Flores ${ }^{a}$ Andrés Mauricio Arroyave-Ramírez ${ }^{a}$ \\ lleana Mackinney-Novelo ${ }^{a}$ Daniel Motola-Kuba a \\ Jose Manuel Ruiz-Morales ${ }^{a}$ Gabriela Alvarado-Luna ${ }^{a}$ \\ Fredy Chable-Montero ${ }^{b}$ \\ ancology Medicine Department, Comprehensive Oncology Center "Diana Laura Riojas \\ de Colosio", Medica Sur Clinic \& Foundation, Mexico City, Mexico; 'bervice of Anatomical \\ Pathology, Medica Sur Clinic \& Foundation, Mexico City, Mexico
}

\section{Keywords}

Cardiac tamponade $\cdot$ Arterial leiomyosarcoma $\cdot$ Pulmonary artery $\cdot$ Pleomorphic

leiomyosarcoma $\cdot$ Chemotherapy

\section{Abstract}

Soft tissue sarcomas represent $<1 \%$ of all neoplasms. Leiomyosarcomas comprise only $5-7 \%$ of cases, and only $2 \%$ of these are vascular. Vascular leiomyosarcomas are extremely rare and represent only $0.001 \%$ of all neoplasms, the venous type being up to 5 times more frequent. Arterial leiomyosarcomas most frequently affect the great vessels, being fatal in most cases. In the reported cases of arterial leiomyosarcomas, the most frequently affected site is the pulmonary artery. We present the clinical case of 2 patients (a 42-year-old woman and a 36-year-old man) with a diagnosis of arterial pleomorphic leiomyosarcoma that conditioned cardiac tamponade as the initial manifestation. As it is an exceptionally rare neoplasm and with few cases reported in the literature, it is important to identify and describe this pathology which, due to the impossibility of offering surgical treatment, represents a therapeutic challenge.

\section{Karger $\stackrel{2}{\circ}$}




\section{Introduction}

Leiomyosarcoma is a smooth muscle tumor; it represents about $10 \%$ of soft tissue sarcoma. Usually, it separates into 3 distinct types such as cutaneous, soft tissue, and big vessels, and the last one is only $<2 \%$. It is most prevalent in veins than arteries, approximately 5 times; it includes 36-60\% inferior vena cava and then the renal, iliac, and femoral vein with $5.6 \%$ [1]. On the other side considering the frequency of arteries, the most affected is the pulmonary artery, according to Malone et al. [2].

The debut of a neoplasm as a clinical picture of cardiac tamponade is a very infrequent event and moreover because a vascular leiomyosarcoma is extremely rare and represents only $0.001 \%$ of all neoplasms [3]. Arterial leiomyosarcomas most frequently affect the great vessels, being fatal in most cases. Specifically, primary pulmonary artery leiomyosarcomas are unique cases, and many a times, they are confused with other pathologies [4].

We present the clinical case of 2 patients with a diagnosis of intracardiac arterial leiomyosarcoma that conditioned cardiac tamponade as the initial manifestation. Since it is an exceptionally unusual neoplasm and with few cases reported in the literature, it represents a therapeutic challenge.

\section{Case Report: 1}

A 42-year-old woman with no significant history came to the emergency room in April 2020 with dyspnea, tachypnea, chest discomfort, peripheral edema, and fatigability. On physical examination, she presented low arterial blood pressure, sinus tachycardia, jugular venous pressure elevated, and muffled heart sounds, for which extension studies were carried out, finding a left mediastinal tumor with extra-pleuropulmonary extension and left atrioventricular tumor attachment in CT. Echocardiogram reported pseudo-alterations in mobility due to extrinsic compression by a $7.7 \times 11 \mathrm{~cm}$ tumor, which compressed the inferolateral, lateral wall of the left ventricle, pericardial effusion, the echo-free space was $22 \mathrm{~mm}$ at its greatest width, type II diastolic function, left ventricular ejection fraction of 58\%, very dilated right ventricle, tricuspid annular plane systolic excursion of $22 \mathrm{~mm}$, and pulmonary artery systolic pressure of $75 \mathrm{~mm} \mathrm{Hg}$ (Fig. 1).

Due to alterations in cardiac contractility, a partial resection of the lesion in its intracardiac portion and pericardiocentesis of $500 \mathrm{~mL}$ was performed. The pathology findings were pleomorphic leiomyosarcoma grade 3, arterial type and immunohistochemistry with desmin positive in $70 \%$ and CD31 and CD34 negative (Fig. 2).

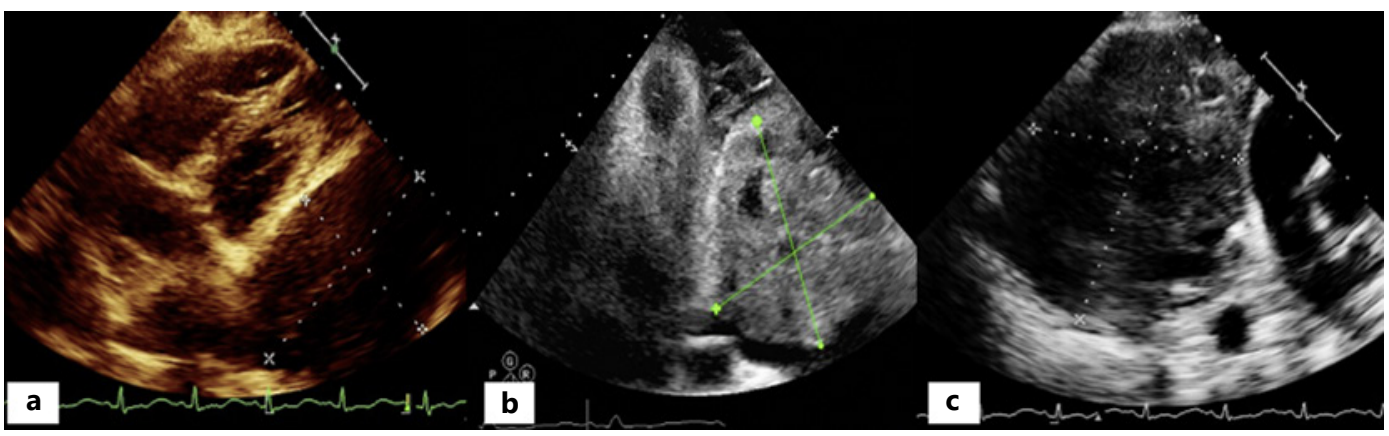

Fig. 1. a-c Echocardiogram reported pseudo-alterations in mobility due to extrinsic compression by a $7.7 \times$ $11 \mathrm{~cm}$ tumor, which compressed the inferolateral, lateral wall of the left ventricle. 


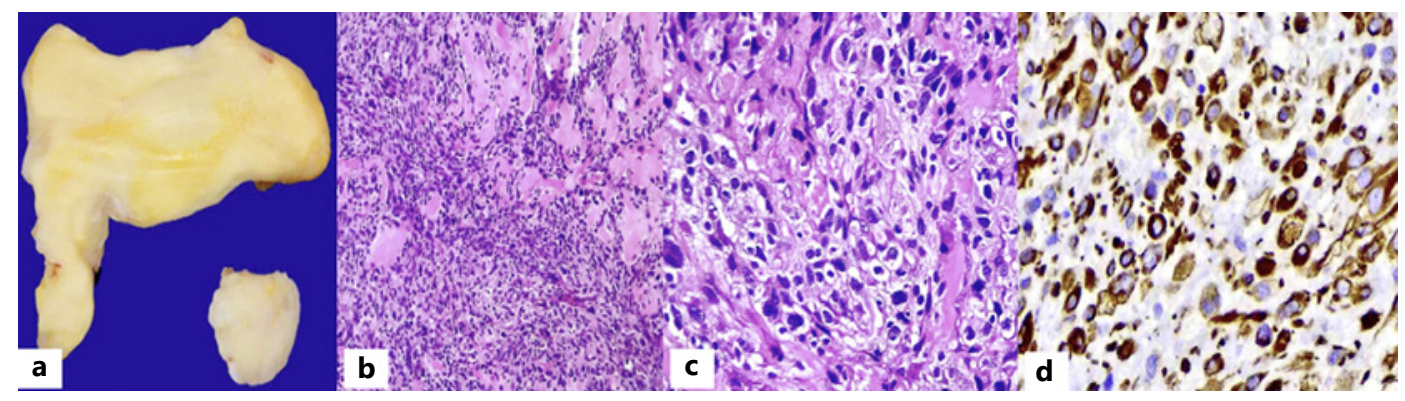

Fig. 2. a Partial resection of the lesion in its intracardiac portion of $4.6 \times 2.4 \times 1.3 \mathrm{~cm}$. The pathology findings were pleomorphic leiomyosarcoma, arterial type, poorly differentiated, and immunohistochemistry with CD31 and CD34 negative (b, c) and desmin positive in $70 \%(\mathbf{d})$.

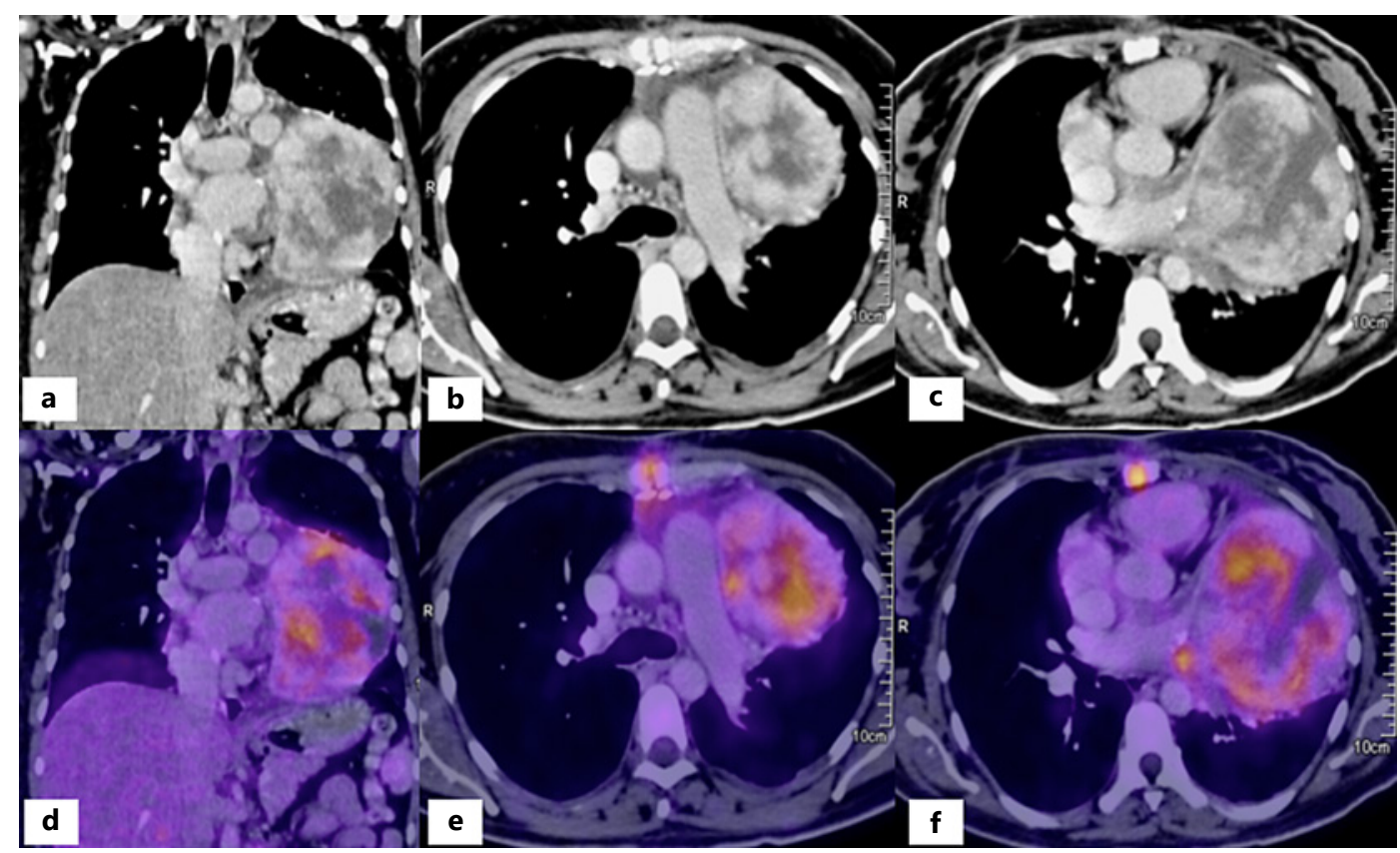

Fig. 3. a-f Fluorodeoxyglucose positron emission tomography/computed tomography shows left paracardiac mediastinal mass enlargement.

Because it was an unresectable tumor, neoadjuvant therapy with gemcitabine $900 \mathrm{mg} / \mathrm{m}^{2}$ on days 1 and 8 and docetaxel $75 \mathrm{mg} / \mathrm{m}^{2}$ on day 1, every 3 weeks, was administered from June to August (received 4 cycles). However, she poorly tolerated chemotherapy presenting cytopenias that required hemocomponent transfusion and granulocyte colony-stimulating factor. Fluorodeoxyglucose positron emission tomography/CT (FDG-PET/CT) was performed in August 2020 showing disease progression (left paracardiac mediastinal mass enlargement and lytic lesion in L1 spinous process (Fig. 3).

Fifteen days later, she presented upper gastrointestinal bleeding, so endoscopy was performed with evidence of a Forrest III ulcer in the antrum and erosions with layer hemorrhage in the duodenum and colonoscopy with GIII hemorrhoidal disease complicated with hemorrhage. A hemorrhoidectomy was carried out. Afterward, she presented clinical deterioration with increased right ventricle filling pressures and severe pulmonary hypertension 
by echocardiogram. Despite multidisciplinary management, she deteriorated, so the medical team decided palliative care. The patient died 2 days later.

\section{Case Report: 2}

A 36-year-old man presented with no relevant history. He began suffering from May 2011 with a dry cough, dyspnea, and later paroxysmal nocturnal dyspnea, asthenia, and adynamia. In October 2011, he presented syncope after coughing and dyspnea for which he went to the emergency room. On physical examination, he presented low arterial blood pressure, tachycardia, tachypnea, chest discomfort, jugular venous pressure elevated, and muffled heart sounds. A transthoracic echocardiogram was performed where the following was reported: pericardial effusion, echo-free space was $25 \mathrm{~mm}$ at its greatest width, heterogeneous left atrial tumor measuring $59 \times$ $40 \mathrm{~mm}$, LVEF 73\%, PSAP $87 \mathrm{~mm}$ Hg, moderate mitral and tricuspid regurgitation, and moderate dilatation of the right chambers. Cardiac MRI subsequently showed a heterogeneous mass of $80 \times$ $50 \times 40 \mathrm{~mm}$ within the left atrium rising from and invading the interatrial septum. It extended into the atrioventricular junction, involving the anterior mitral valve leaflet. In November 2011, a left atrial tumor resection and mitral valve replacement were performed using a 27-mm Carpentier Edwards Perimount prosthesis. Macroscopically, a large homogeneous tissue mass involving the inferior and posterior left atrial walls and adherent to the anterior mitral valve leaflet was found. The diagnosis of high-grade vascular type leiomyosarcoma was performed by pathology. Immunohistochemistry of tumor was actin positive and desmin negative (Fig. 4).

MRI reported metastasis to the central nervous system, and FDG-PET/CT reported bone metastasis (thoracic 12 and lumbar 1 ). The patient received systemic palliative chemotherapy with gemcitabine $800 \mathrm{mg} / \mathrm{m}^{2}$ on days 1 and 8 and docetaxel $75 \mathrm{mg} / \mathrm{m}^{2}$ on day 1 every 3 weeks and received 2 cycles for 6 weeks postoperatively. Our patient died 5 months after presentation.

\section{Discussion}

The estimated incidence of pulmonary artery leiomyosarcoma is between 0.001 and $0.03 \%$, being extremely rare and highly lethal [5]. Primary leiomyosarcomas of the lung are extremely rare and may arise from the smooth muscles of the pulmonary interstitium, bronchi, and blood vessels [6].

Leiomyosarcomas usually occur during the 6th decade of life or later, predominantly in men; however, pulmonary artery leiomyosarcomas are more frequent in women $(2: 1)$ versus men, and the mean age at diagnosis is 52 years $[7,8]$.

Primary cardiac tumors are extremely rare with an incidence of $0.02 \%$ per person-year. The majority of primary cardiac tumors is benign myxomas with around a $10 \%$ chance of being malignant. Autopsy studies indicate that leiomyosarcomas constitute $<1 \%$ of all malignant cardiac neoplasms and only 8-9\% of all cardiac sarcomas. Cardiac leiomyosarcoma typically presents in the fourth decade [1-8].

Usually, the tumor growth is endovascular to exovascular, and we can stage it in 3 different forms: nonocclusive, occlusive, and terminal. The nonocclusive is asymptomatic, and the diagnosis is clinical suspicion; the occlusive stage is asymptomatic in the majority, but some patients have phlebitis and unilateral edema. The last stage is diagnosis in $10 \%$ of the patients [9]. Other classification is based on the origin of the growth; the intima growth inside the lumen of the vessel, and it extends long the vessel or like polypoid mass, and the clinical symptoms and sign are correlated by the obstruction grade; media/adventitia growth; extension is extramural or intramural; and the presentation depends on the growth form [1,5-9].

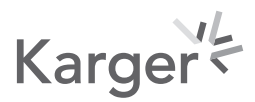




\section{Case Reports in Oncology}

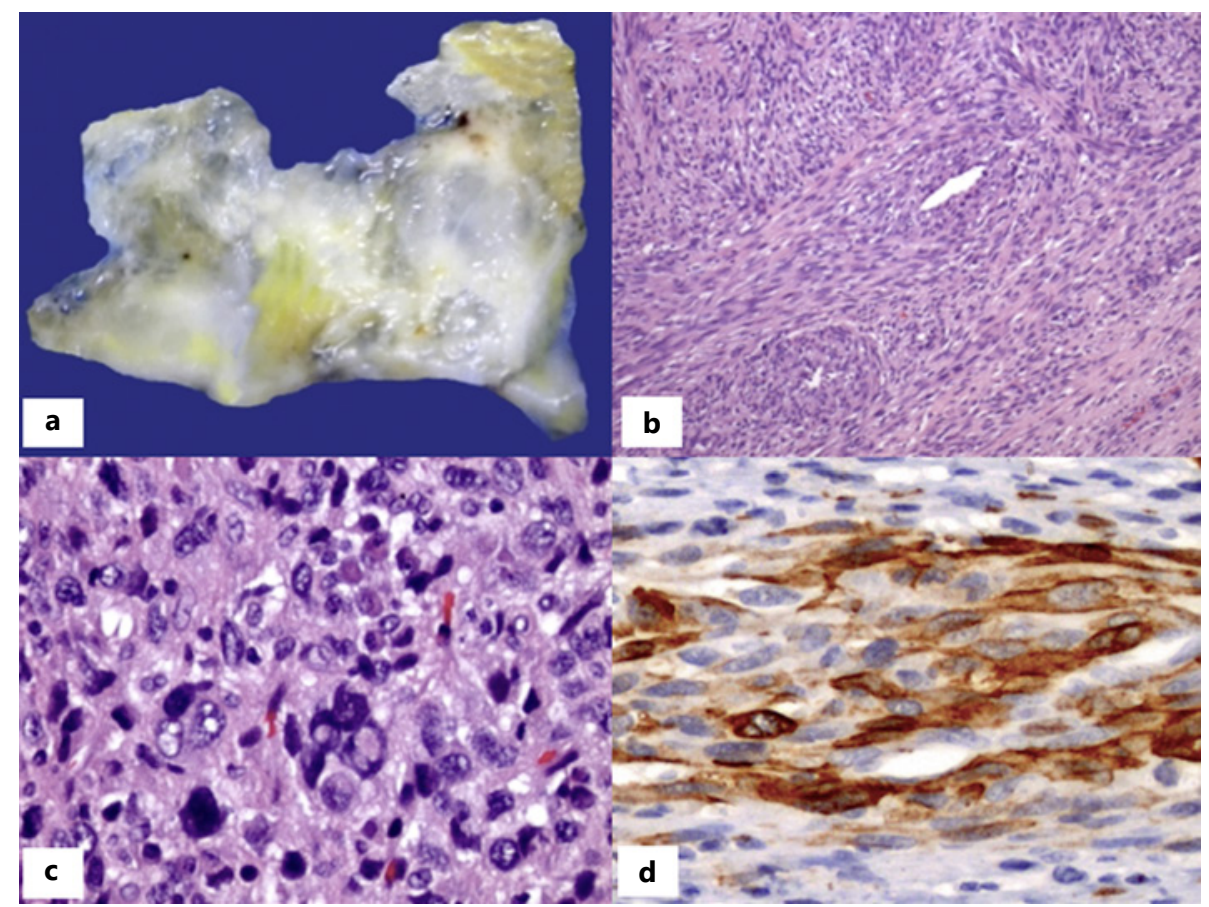

Fig. 4. a An irregular and myxoid tumor, yellow-grayish, that measured $8 \mathrm{~cm}$ in the greatest dimension. $\mathbf{b}$ Smooth muscle bundles that show predominant perivascular distribution. c Epithelioid foci with marked pleomorphism and mitotic figures. $\mathbf{d}$ The tumor was actin positive. The diagnosis of high-grade vascular type leiomyosarcoma was performed.

In the scarce information reported in the literature, arterial leiomyosarcomas affect more frequently the pulmonary artery trunk in $56 \%$ of the patients, but can also occur in the right and left main pulmonary arteries, the pulmonary valve, and the right ventricular outflow tract [10]. Transmural spread of the tumor into adjacent lung parenchyma, bronchi, or lymph nodes occurs in approximately $50 \%$ of cases and may extend to the myocardium and mediastinum. Systemic metastases are usually found in approximately $20 \%$ of cases $[2,3,6,8-11]$.

Overall, right atrial neoplasms are more likely to be malignant than left atrial tumors which are usually myxomas. However, as our cases illustrated, leiomyosarcoma favors the left atrium and tends to invade the pulmonary veins and mitral valve [2, 6-11].

The main symptoms are chest pain, dyspnea, and signs of right-sided heart failure, usually misdiagnosed as a venous thromboembolic event $[7,8]$. It is important to acknowledge when this clinical presentation persists even after anticoagulation therapy in a patient without risk factors for thromboembolism [8,9]. Additional symptoms have been described such as palpitations, syncope, weight loss, and cough $[6,9,12,13]$. However, when they present with pericardial effusion, they may present clinical characteristics of cardiac tamponade (dyspnea, tachypnea, peripheral edema, angina, low arterial blood pressure, tachycardia, tachypnea, chest discomfort, jugular venous pressure elevated, and muffled heart sounds), like our cases.

Traditionally, echocardiography has been the primary imaging modality to diagnose, differentiate, and delineate intracardiac masses. The accessibility, reliability, and cost effectiveness of echocardiography continue to make this the initial investigation of choice [9-12]. Although cardiac tamponade is a clinical diagnosis, 2-dimensional and Doppler echocardiography play major roles in the identification of pericardial effusion and in assessing its hemodynamic significance (chamber collapse, respiratory variation in volumes and flows, and dilated inferior vena cava), and pericardial effusion, the echo-free space $>0 \mathrm{~mm}$ at its greatest width, 
is considered moderate effusion $(100-500 \mathrm{~mL})$. Transthoracic echocardiography is a widely available screening examination and remains the basic test for cardiac tumors. Extended analysis requires transesophageal echocardiography, particularly useful for visualization of changes within the left atrium, small tumor, or infiltration beyond the pericardium. What we were able to observe in these 2 patients were mainly tumor masses with extrinsic compression of the cardiac chambers, which altered cardiac mobility, and data on cardiac compromise at an earlier age in previously healthy patients.

Imaging studies can help to establish the diagnosis, even though a pulmonary arterial leiomyosarcoma and venous thromboembolic disease can present similar findings $[9,14]$. Vascular images like ultrasound Doppler, computerized angiotomography, and arteriography show filling defect, but they cannot differentiate between embolic process and thrombotic tumor origin; for this reason, we need to do a magnetic resonance of the probable origin of tumor and chest and abdominal and pelvis computerized tomography to staging disease [15].

MRI shows areas of hemorrhage with or without necrosis. Increasingly, cardiac CT and cardiac MRI are used to provide complementary information. When FDG-PET is done, it can be helpful for the differential diagnosis. It shows increased uptake in the tumoral area and metastases sites $[12,16,17]$.

However, if we want to do the diagnosis, we need a fine needle biopsy with a diagnostic performance of $60-95 \%$ or trucut biopsy with diagnostic performance $93 \%$. Some experts prefer excision biopsy in tumor with easy access because with the other procedures, wide variability exists [18].

The diagnosis can be confirmed by pathological and immunohistochemical examination. In the histology of the leiomyosarcomas, we can identify malignant shape cells and nuclei like cigarette, which are arranged in interlocking bundles. The bundles of filaments contain body dense of pinocytic vesicles. When actin, smooth muscle actin, and desmin immunohistochemical results are positive, it is presumed that the tumor originates from smooth muscle. We can auxiliary the other markers when we have the idea that is secondary to metastases of the other organs like S100 y HBM45 (melanoma); chromogranin, synaptophysin, and specific enolase of nerve cells (neuroendocrine tumor); and CD20 and CD30 (hematolymphatic tumor) $[19,20]$.

The staging is according to the American Joint Committee on Cancer (AJCC), based on histologic grade, size and depth, involved nodes, and metastases at distance. The histological grade is according to the FNLCC (Federation Nationale des entres de Lutte Contre le Cancer), and it is based on cellularity grade, differentiation, pleomorphic, necrosis, and mitosis [6-20].

The treatment is multidisciplinary with medical oncologist, surgery, and vascular surgery. The objective is the resection of the tumor with wide margins $(2-3 \mathrm{~cm})$ with a survival at 5 years of $80-90 \%$. However, wide and complete resection is not possible in the majority of the patients, and the survival is only $25-49 \%$. The usefulness of adjuvant radiotherapy is not clear, and in small retrospective studies or cases series, doxorubicin or dacarbazine is selected. According to current data, anthracycline-based chemotherapy (adriamycin/doxorubicin + ifosfamide) is recommended. In the case of anthracycline resistance, sorafenib or trabectedin can be used in soft tissue sarcomas. However, sorafenib gives only a 6-month progression-free rate of about $20 \%$ in patients with angiosarcoma. The regimen with gemcitabine + docetaxel (or paclitaxel) is considered in those patients with contraindication to anthracyclines, especially in those with poor cardiac function [2-13, 21].

Despite the high risk of mortality due to cardiac tamponade and an extremely aggressive disease, in which a complete resection of the tumor was not achieved or with metastasis at diagnosis, an overall survival of approximately 5 months was achieved with gemcitabine +

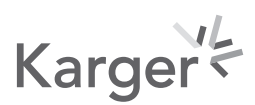


docetaxel-based chemotherapy. It is a diagnostic and therapeutic challenge due its location and impossibility to perform the excision of the lesion. Survival range up to 36 months after treatment has been reported. Despite this, they are uncommon highly aggressive neoplasms with 1-year survival estimated at $20 \%$ from the onset of symptoms [22].

Median overall survival is 16 months, related to the possibility of complete resection of the lesion, so an attempt to excise the lesion should be done. In patients without surgery, the overall survival is about 1.5 months. Most of the patients die because of right-sided heart failure. Approximately, $60-70 \%$ of the patients develop metastases to the lungs [23-25].

\section{Conclusion}

Pulmonary arterial pleomorphic leiomyosarcoma is very rare. To date, there are $<150$ cases reported in the English literature. The treatment is multidisciplinary with medical oncologist, surgery, and vascular surgery and has poor prognosis.

\section{Acknowledgments}

The authors thank the Medica Sur Hospital, for their support in data collection.

\section{Statement of Ethics}

Because both patients died, the informed consent was signed by their parents. The informed consent of the first patient (female) was signed by her father. The second case corresponded to a man who unfortunately died, so the informed consent was signed by his father. Both parents gave their informed consent in writing to publish their children's case (including the publication of images). The study is exempt from ethics committee approval because only a review of the clinical record was carried out, and it was not an experimental study.

\section{Conflict of Interest Statement}

The authors have no conflicts of interest to declare.

\section{Funding Sources}

We did not receive any funding for the preparation of this case report.

\section{Author Contributions}

Ricardo Fernández-Ferreira contributed to the conception of the case, analysis, and critical revision of the content, as well as the final approval of the version to be published. Emilio Conde-Flores, Pamela Denisse Soberanis-piña, Andrés Mauricio Arroyave-Ramírez, Ileana Mackinney-Novelo, Daniel Motola-Kuba, and Jose Manuel Ruiz-Morales contributed to the critical revision of the content, as well as the final approval of the version to be published.

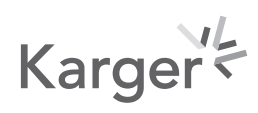


Fernández-Ferreira et al.: Arterial Leiomyosarcoma

Fredy Chable-Montero carried out an exhaustive review of the histopathological characteristics of cancer and analysis of the article. All authors agree to be responsible for all aspects of the job to ensure that questions related to the accuracy or completeness of any part of the job are properly investigated and resolved.

\section{Data Availability Statement}

Data supporting the findings of this study are openly available in the clinical file of Medica Sur Hospital, with registration number: 1000252322. Additional inquiries can be directed to the corresponding author.

\section{References}

1 Weiss SW. Smooth muscle tumors of soft tissue. Adv Anat Pathol. 2002;9(6):351-9.

2 Malone MD, Kerr K, Kavanah M, Menzoian JO. Primary leiomyosarcoma of the abdominal aorta. J Vasc Surg. 1996;24(3):487-93.

3 Sardenberg RA, Cangnaci Neto R, Cavalcanti F, Younes RN. High-grade primary pulmonary leiomyosarcoma. Einstein. 2011;9:523-6.

4 Adeli SH, Nemati B, Jandaghi M, Riahi MM, Hosseinzadeh F, Salarvand F. Pulmonary hypertension due to a pulmonary artery leiomyosarcoma: a case report. Case Rep Pulmonol. 2013;2013:160619.

5 Hoggemeier A, Semik M, Fallenberg EM, Scheld HH. Leiomyosarcoma of the pulmonary artery-a diagnostic chameleon. Eur J Cardiothorac Surg. 2001;20:1049-51.

6 Demirci NY, Naurzvai N, Kirbaş I, Akyürek N, Gürsel G, Öztürk C. Pulmonary artery leiomyosarcoma: a clinical dilemma. Lung India. 2018;35(2):164-7.

7 Anderson MB, Kriett JM, Kapelanski DP, Tarazi R, Jamieson SW. Primary pulmonary artery sarcoma: a report of six cases. Ann Thorac Surg. 1995;59:1487-90.

8 Akram K, Silverman ME, Voros S. A unique case of pulmonary artery leiomyosarcoma. J Natl Med Assoc. 2006; 98:1995-7.

9 Kim JH, Gutierrez FR, Lee EY, Semenkovich J, Bae KT, Ylagan LR. Primary leiomyosarcoma of the pulmonary artery: a diagnostic dilemma. Clin Imaging. 2003;27:206-11.

10 Muganlinskaya N, Guzman A, Dahagum C, Selinger SR. When a pulmonary embolism is not a pulmonary embolism: a rare case of primary pulmonary leiomyosarcoma. J Community Hosp Intern Med Perspect. 2015;5:1-3.

11 Sakpal SV, Mehta R, Babel N, Chamberlain RS. Peripheral artery leiomyosarcoma. J Vasc Surg. 2009;49(1):217-21.

12 Li B, Zhang Y, Cai L, Hou J, Shi H. Primary pulmonary artery sarcoma differentiated from pulmonary thromboembolism by ventilation-perfusion scan. Long survival of the patient. Hell J Nucl Med. 2015;18(2):166-8.

13 Jing R, Tang H, Shen K, Luo J, Zhou X. A rare case of primary pulmonary artery myofibroblastic sarcoma: imaging and pathologic features. Ann Vasc Surg. 2014;28(2491):491-7.

14 Hoffmeier A, Semik M, Fallenberg EM, Scheld HH. Leiomyosarcoma of the pulmonary artery: a diagnostic chameleon. Eur J Cardiothorac Surg. 2001;20:1049-51.

15 Abed R, Abudu A, Grimer RJ, Tillman RM, Carter SR, Jeys L. Leiomyosarcomas of vascular origin in the extremity. Sarcoma. 2009;2009:385164.

16 Kessler A, Son H. Pulmonary artery angiosarcoma on 18F-FDG PET/CT masquerading as pulmonary embolism. Clin Nucl Med. 2015;40:82-4.

17 Jing R, Tang H, Shen K, Luo J, Zhou X. A rare case of primary pulmonary artery myofibroblastic sarcoma: imaging and pathologic features. Ann Vasc Surg. 2014;28(2491):491-7.

18 Saglik Y, Icli F, Uluoglu O, Isiklar ZU. Leiomyosarcoma of the great saphenous vein. Int Orthop. 1992;16(2):185-7.

19 Arnold LM 3rd, Burman SD, O-Yurvati AH. Diagnosis and management of primary pulmonary leiomyosarcoma. J Am Osteopath Assoc. 2010;110:244-6.

20 Coli A, Parente P, Bigotti G. Pulmonary artery sarcoma: an insidious tumor still diagnosed too late. Analysis of the literature and report of a case. J Exp Clin Cancer Res. 2007 Mar;26(1):151-6.

21 Marle AG, Bronkhorst MW, Brouwers MA. Leiomyosarcoma of the great saphenous vein: a case report and review of the literature. Sarcoma. 2004;8(4):135-9.

22 Stella F, Davoli F, Brandolini J, Dolci G, Sellitri F, Bini A. A pulmonary artery leiomyosarcoma successfully treated by right pneumonectomy. Asian Cardiovasc Thorac Ann. 2009;17(5):513-5.

23 Shen W, Chen J, Wei S, Wang X, Li X, Zhou Q. Primary pulmonary leiomyosarcoma. J Chin Med Assoc. 2014;77:49-51.

24 D'Andrea N, Romano V, Mattioli F, Candia S, Montanari A, Guidi M, et al. Pulmonary artery leiomyosarcoma with thyroid metastases. Monaldi Arch Chest Dis. 2003;59:304-7.

25 Italiano A, Toulmonde M, Stoeckle E, Kind M, Kantor G, Coindre JM, et al. Clinical outcome of leiomyosarcomas of vascular origin: comparison with leiomyosarcomas of other origin. Ann Oncol. 2010;21(9):1915-21.

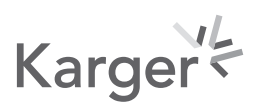

